\title{
Some Aspects of the Genetics of Methionineless Mutants of Salmonella typhimurium
}

\author{
By D. A. SMITH \\ Microbiological Research Establishment, Porton Down, Salisbury, Wiltshire \\ SUMMARY
}

Forty-six methionineless mutants of Salmonella typhimurium were arranged in three phenotypic groups according to their growth responses to potential precursors of methionine. The results of syntrophism tests led to the recognition of two more phenotypic groups and permitted the arrangement in sequence of the metabolic steps in which mutants of each of the five groups were deficient. Transduction experiments indicated that each of these groups comprised mutants whose sites of mutation were closely linked within a complex locus. Attempts to map the sites of mutation of 12 mutants within one locus were unsuccessful. One of the mutants, probably a deletion, failed to recombine with the other 11 and behaved differently from them in linked transduction. A group of three and one pair of the 11 mutants could not be separated by recombination. Linkage was detected by transduction between only 2 of the 5 loci; these were concerned with non-sequential steps in the biosynthesis of methionine. No linkage was detected between the methionine loci and any of a number of other loci, including those controlling the biosyntheses of eysteine and tryptophan. These results were only partly in accordance with a previously suggested linkage map.

\section{INTRODUCTION}

The transfer of genetic fragments between strains L'T-2 and LT-7 of Salmonella typhimurium and their derivatives by the temperate phage PLT-22 (transduction) was first reported by Zinder \& Lederberg (1952). This was later defined as a system of common transduction in that, apparently, any marker could be transferred (Clowes, 1960). Since 1952, this system has been applied to the study of fine genetic structure by Demerec and his co-workers who used large numbers of auxotrophic, fermentative and antibiotic-resistant mutants. The genetic subdivisibility of the functional gene (locus) has been demonstrated (Hartman, 1956; Demerec \& Hartman, 1956). Genes concerned with related functions were often found to be closely linked (Demerec, 1956a). A complementation test (abortive transduction) has permitted the definition of a functional unit (Demerec \& Ozeki, 1959). Studies of the fine structure of certain loci have led to the construction of detailed linkage maps revealing, for example, the probable occurrence of deletions (Demerec, Blomstrand \& Demerec, 1955; Clowes, 1958; Hartman, Loper \& Serman, 1960) and leading to an understanding of the nature of suppressor activity (Yura, 1956 $b$; Gots, 1956; Howarth, 1958). Evidence has also been obtained that recombination occurs in transduction by crossing-over (Demerec \& Demerec, 1955) and a copy-choice model has been suggested (Demerec, Goldman \& Lahr, 1958). Fragmentation of the donor 
chromosome in the production of fragments incorporated into transducing phage is probably not random (Ozeki, 1959).

Previous work with 32 methionineless (met) mutants led to the recognition of 5 loci (met $A$, met $B$, metC, met $E$, met $F)$ based primarily on the frequency of transduction between mutants (Glover, 1955, 1956). Growth responses to precursors of methionine, syntrophism tests and chromatographic analyses of substances accumulated by representative strains indicated that each locus comprised mutants with identical biochemical properties which were different from those of mutants of the other loci (Glover, 1956, 1958). Linkage studies (Glover, 1955, 1956) led to the construction of a map in which all the met loci, the 4 linked tryptophan loci (Demerec \& Hartman, 1956) and 4 loci controlling cysteine synthesis were linked together.

It was originally intended to examine whether each of a number of newly-isolated met mutants could be assigned to an appropriate met locus and to investigate the fine structure of one of these loci by transduction. Nineteen new mutants and 27 of those previously used were classified and the fine structure of a locus containing 12 mutants examined. Incidental to this work was a re-investigation of linkage between the met loci and between these and other loci.

The system of nomenclature used is that of Demerec (1956b) and elaborated in the Microbial Genetics Bulletin, 1958, No. 16. Each type of mutant is given a symbol, e.g. met for methionine requirement, a number according to its chronological isolation, e.g. met-2, and a letter (e.g. $E$ ) if subsequently assigned to a locus, e.g. metE-2. In transduction, there is a transfer of genetic fragments from one strain of bacterium (donor) from which phage is prepared, to another strain (recipient) infected with this phage. A cross is written: recipient (bacterium) $(x)$ donor (phage), e.g. metE-2( $\times)$ metC-30, to designate the transduction resulting from the infection of metE-2 bacterium with phage prepared on metC-30. The terminology is in accordance with Demerec (1956b). A 'gene' is considered to occupy a linear section of a chromosome called a 'locus' concerned with the control of one function. This linear section is subdivisible into units termed 'sites' separable by crossing-over. 'Allele' is defined as one of several forms in which a gene exists. Two alleles are 'non-identical' when due to mutation at different sites, 'identical' when homologous sites are involved.

\section{METHODS}

Organisms. The wild-type strain LT-2 of Salmonella typhimurium (Zinder \& Lederberg, 1952) and 46 different methionineless (met) mutants of it or of other auxotrophic derivatives from it, were used (Table 1). All the mutants except metC-30 were obtained (in the Department of Genetics, Carnegie Institution of Washington, Cold Spring Harbor, N.Y., U.S.A.), either as spontaneous or ultraviolet-induced mutants by using the penicillin selection technique (Davis, 1948; Lederberg \& Zinder, 1948). MetC-30 was a spontaneous mutant isolated by Dr N. D. Zinder (personal communication). Twenty-seven mutants (met-2 to met-45 inclusive) had been used by Glover $(1955,1956,1958)$ in his biochemical and genetical studies. The remaining 19 strains (met-47 to met-68 inclusive) were newly isolated.

Stock cultures of these strains were maintained as stabs in $0.7 \%$ Bacto agar in small corked tubes which had been incubated overnight, sealed with paraffin wax and then kept at room temperature. Viability was retained during $2 \frac{1}{2}$ years. For day to 
day use, strains were kept at $0-5^{\circ}$ on nutrient agar slopes and subcultured every 6 weeks. Their nutritional requirements were tested periodically by an auxanographic technique.

Table 1. Methionineless (met) mutants of Salmonella typhimurium

\begin{tabular}{|c|c|c|c|c|c|}
\hline Mutant & Group & Other markers & Mutant & Group & Other markers \\
\hline met- $6^{*}$ & $\boldsymbol{A}$ & . & $m e t-45$ & $\boldsymbol{B}$ & adeE-11 \\
\hline$-7 *$ & $\boldsymbol{A}$ & cys $A-21$ & -49 & $B$ & - \\
\hline$-10 *$ & $A$ & cys $B-12$ & -66 & $\boldsymbol{B}$ & . \\
\hline$-15^{*}$ & $\boldsymbol{A}$ & . & -30 & $C$ & . \\
\hline-18 & $A$ & . & -32 & $C$ & adth $A-2$ \\
\hline-19 & $A$ & $\operatorname{try} A-8$ & -35 & $C$ & adth $A-2$ \\
\hline-21 & $\boldsymbol{A}$ & $\operatorname{try} A-8$ & -37 & $C$ & adth $A-2$ \\
\hline-22 & $A$ & $\operatorname{try} B-2$ & -50 & $C$ & . \\
\hline-40 & $A$ & adthA-2 & -52 & $C$ & . \\
\hline-43 & $A$ & adeE-11 & -56 & $C$ & . \\
\hline-44 & $A$ & adeE-11 & -57 & $C$ & . \\
\hline-48 & $A$ & . & -58 & $C$ & . \\
\hline-53 & $A$ & . & -65 & $C$ & - \\
\hline-54 & $A$ & . & $-2^{*}$ & $\boldsymbol{E}$ & cysC-7 \\
\hline-55 & $A$ & . & -47 & $\boldsymbol{E}$ & . \\
\hline-16 & $\boldsymbol{B}$ & $\operatorname{cys} B-16$ & -51 & $\boldsymbol{E}$ & . \\
\hline-17 & $\boldsymbol{B}$ & cysB-16 & -60 & $\boldsymbol{E}$ & . \\
\hline-20 & $\boldsymbol{B}$ & $\operatorname{tr} y A-8$ & -61 & $E$ & . \\
\hline-23 & $B$ & . & -68 & $E$ & $\cdot$ \\
\hline-33 & $B$ & adthA-2 & -31 & $\boldsymbol{F}$ & adth $A-2$ \\
\hline-34 & $B$ & $\operatorname{adth} A-2$ & $-31 r * *$ & $F$ & . \\
\hline-36 & $B$ & $\operatorname{adth} A-2$ & $-59 * * *$ & $F$ & . \\
\hline-39 & $\boldsymbol{B}$ & adth $A-2$ & $-64 * * *$ & $F$ & . \\
\hline-41 & $\boldsymbol{B}$ & adeE-11 & - & . & - \\
\hline
\end{tabular}

ade $=$ adenineless $;$ adth $=$ adenine and thiaminless $;$ cys $=$ cysteineless $;$ try $=$ tryptophaniess; * Obtained after ultraviolet irradiation; $* *$ an $a d t h^{+}$reversion derived from metF-31; *** high frequency of reversion.

In all experiments incubation was at $37^{\circ}$. All liquid cultures were aerated either by bubbling air through them or by agitation at $60-70 \mathrm{rev} . / \mathrm{min}$. in tubes held at an angle of $30^{\circ}$. Sedimentation by centrifugation was at approximately $2000 \mathrm{~g}$ for 15-20 min.; washing was carried out with $0.5 \%(w / v) ~ \mathrm{NaCl}$ solution or $33 \mathrm{~mm}$ phosphate buffer $\left(\mathrm{KH}_{2} \mathrm{PO}_{4}\right)$ adjusted to $\mathrm{pH} 7$ with $\mathrm{NaOH}$.

Phage. The temperate phage PLT-22 (Zinder \& Lederberg, 1952) was used for transduction. Donor lysates were prepared by the infection, at a multiplicity of $0 \cdot 05-0 \cdot 1$, of $\log$-phase cultures of bacteria with phage initially propagated on wildtype LT-2. Incubation was continued for $6 \mathrm{hr}$. Phage was then sedimented by centrifugation at $78,000 \mathrm{~g}$ for $1 \mathrm{hr}$. The deposited phage was resuspended in $\frac{1}{20}$ th volume T 2 buffer (adsorption medium; Hershey \& Chase, 1953) and re-centrifuged at normal speed, the opalescent supernatant fluid then being retained. The phage preparations were sterilized by shaking with $\frac{1}{10}$ th volume of chloroform for 30 min. at room temperature and then removing the chloroform by bubbling with air at $37^{\circ}$ or room temperature. The layer-plate method (Adams, 1950) was used for phage assay, two separate $1 \times 10^{-10}$ dilutions of each preparation being plated in triplicate or quadruplicate. The titres of the phage suspensions ranged from $3 \times 10^{11}$ to $2 \times 10^{12}$ particles/ml. and were constant during many months of storage at $0-5^{\circ}$.

Transduction. In early experiments, phage from donors was added to overnight 
broth cultures of recipient strains at $37^{\circ}$ at a multiplicity of 5 . After 6 min. adsorption, $0 \cdot 1 \mathrm{ml}$. samples of the mixtures were plated on selective minimal media. Phage and recipient bacterial controls were plated at the same time. The number of prototrophic colonies was counted after incubation up to at least $40 \mathrm{hr}$. The frequency of transduction obtained with met mutants by this method (about 1 per $10^{7}-10^{8}$ donor phage particles) was low compared with that obtained with other auxotrophs of Salmonella typhimurium. A 10-100-fold increase in frequency of transduction with the met mutants as recipients was obtained by adding phage at $4 \times 10^{10}$ particles/ml. to cultures in the middle of the log phase. Cultures of suitable titre were obtained by diluting overnight broth cultures $1 / 20$ in broth, incubating for $2 \mathrm{hr}$., centrifuging and then resuspending in $\frac{1}{4}$ volume of broth. This yielded a culture containing $2-4 \times 10^{9}$ organisms $/ \mathrm{ml}$. The multiplicity of infection was therefore at least 10. In some experiments it was necessary to have plates with 100-200 transduced colonies. After samples had been removed for plating, transduction mixtures were stored at $0-5^{\circ}$ for $24 \mathrm{hr}$. for replating, when required, using new appropriate dilutions.

Media. The nutrient medium used was either 0.8\% (w/v) Difco Beef Peptone in $0.5 \%(\mathrm{w} / \mathrm{v}) \mathrm{NaCl}$ in water or in the tryptic meat digest broth (TMB) made in this Establishment. The addition of agar at $2 \%(\mathrm{w} / \mathrm{v})$ provided a plating medium (TMA). The minimal medium (MM) was that of Davis (see Lederberg, 1950) with the $\mathrm{K}_{2} \mathrm{HPO}_{4}$ and $\mathrm{KH}_{2} \mathrm{PO}_{4}$ concentrations adjusted to $10.5 \mathrm{~g}$. and $4.5 \mathrm{~g}$./ $/$., respectively. Plating medium (MA) was obtained by the addition of agar $(1.5 \%, w / v)$. The following growth factors $(\mu \mathrm{g} . / \mathrm{ml}$.) were added as required: adenine $\mathrm{HCl}, 20$; L-cysteine $\mathrm{HCl}, 80$; DL-homocysteine thiolactone $\mathrm{HCl}, 100$; DL-methionine, 20; thiamin (aneurin) $\mathrm{HCl}, 1$; DL-tryptophan, 20; vitamin $\mathrm{B}_{12}, 0 \cdot 1$. Plating medium $\mathbf{M A}$ was occasionally enriched with $0.01 \%$ or $1.25 \%(v / v)$ broth (eMA and EMA, respectively).

Purity of chemicals. The purity of the preparations of DL-cystathionine $\mathrm{HCl}$, L-cysteine $\mathrm{HCl}$, L-homocystine, DL-homocysteine thiolactone $\mathrm{HCl}$, DL-methionine and vitamin $B_{12}$ used in the growth response experiments was tested by means of one-dimensional paper chromatography with collidine as the solvent (Dent, 1948). The preparations of cystathionine, homocystine, vitamin $\mathrm{B}_{12}$ and methionine were pure; those of cysteine and homocysteine thiolactone were not. The identification of three impurities besides cystine in the cysteine was not attempted. The preparation of homocysteine thiolactone contained homocystine and probably homocysteine; these may have resulted from slow hydrolysis of the thiolactone (Dubnoff, 1952).

\section{RESULTS}

The utilization of methionine precursors on the known pathway of methionine biosynthesis in Neurospora crassa (Horowitz, 1947), and of vitamin $\mathbf{B}_{12}$, known to be utilized by some methionineless mutants of Escherichia coli (Davis \& Mingioli, 1950) was tested by surface inoculation of all mutants to supplemented MA plates as in the normal auxanographic technique (Pontecorvo, 1949). Plates supplemented with different concentrations of cystathionine, cysteine, homocystine, homocysteine thiolactone, methionine or vitamin $\mathbf{B}_{12}$ were inoculated with drops, each containing about $2 \times 10^{4}$ organisms, from phosphate buffer dilutions of washed organisms from overnight broth cultures of each mutant, by using a multiple inoculation 
device (Smith, unpublished) and incubated for $66 \mathrm{hr}$. The growth responses observed were similar to those obtained by Glover (1956, 1958); his nomenclature will be used. All mutants responded to methionine; none to cysteine. Thirty-seven mutants responded to either cystathionine, homocystine or homocysteine thiolactone (met $A+$ metB + metC mutants), of which 10 (later designated metC by syntrophism experiments), grew slowly on MA medium and were considered to be 'leaky'. Six other mutants responded to vitamin $\mathrm{B}_{12}$ (met $\boldsymbol{E}$ mutants) and three others to methionine only (metF mutants). Thus, on the basis of growth responses alone, three phenotypic groups were recognizable, comprising the met $\boldsymbol{A}+$ met $\boldsymbol{B}+$ met $C$, the met $\boldsymbol{E}$ and the met $\boldsymbol{F}$ mutants.

The lowest concentrations of each substance and the times of incubation for maximum growth responses were recorded. Growth responses of mutants (other than the leaky mutants) to methionine $(5-10 \mu \mathrm{g} . / \mathrm{ml}$.$) , homocystine (20 \mu \mathrm{g} . / \mathrm{ml}$.$) ,$ homocysteine thiolactone (50 $\mu \mathrm{g} . / \mathrm{ml}$.) and vitamin $B_{12}(0.001 \mu \mathrm{g} . / \mathrm{ml}$.) were maximal after incubation for $42 \mathrm{hr}$. and to cystathionine (50-100 $\mu \mathrm{g} . / \mathrm{ml}$.) after incubation for $66 \mathrm{hr}$. The leaky mutants responded to methionine, cystathionine, homocystine or homocysteine thiolactone at concentrations of one-half to one-tenth of those necessary for met $A$ and $m e t B$ mutants.

The slow growth response of met $A+$ met $B+$ met $C$ mutants to cystathionine at high concentration was further investigated by chromatographic examination of solutions of the substance in sterile distilled water and MM medium after incubation for $66 \mathrm{hr}$. The former was pure but the latter contained small quantities of two impurities, one of which was homocystine, which might well have been responsible for the growth in the presence of cystathionine. However, the lag in growth response to cystathionine still persisted after inoculation on to MA medium + cystathionine at $50 \mu \mathrm{g} . / \mathrm{ml}$. pre-incubated for $66 \mathrm{hr}$.

\section{Indication of the sequence of metabolic blocks with which each group of mutants was associated}

This was determined by means of syntrophism tests. Two methods gave readily reproducible results.

Parallel streaking. Loop inocula from saline dilutions (1/1000) of washed overnight broth cultures of mutants were streaked $0.5-1 \mathrm{~cm}$. apart on EMA medium, each pair of inocula being isolated either in separate $4 \mathrm{~cm}$. diameter Petri dishes or on sectors of solid medium in $9 \mathrm{~cm}$. diameter Petri dishes. Control streaks of homologous pairs and mutants alongside wild-type were always made. Syntrophism was indicated by relatively denser growth of the edge of a streak adjacent to one releasing a diffusible compound (accumulant) and was visible after incubation for $60 \mathrm{hr}$. but more clearly after $85-90 \mathrm{hr}$.

All paired combinations of the strains met $A-6, B-16, C 30, E-2$ and $F-31$, representative of Glover's 5 groups, were tested in this way. Strain metC-30 fed strains met $A-6$ and $B-16$ but not strains metE-2 or $F-31$. No other feeding was clearly demonstrated. The other 9 leaky strains behaved like strain metC-30 in similar experiments. These results indicated that the 10 leaky mutants comprised a fourth phenotypic group (met $C$ ) and that the metabolic blocks in the biosynthesis of methionine associated with the $m e t A$ and $B$ mutants preceded, and those of the metE and $F$ mutants followed, those of metC. 
Replica plating. Inocula $(0 \cdot 1 \mathrm{ml}$.) from washed overnight broth cultures of strains met $A-15, B-23, C-30, E-47$ and $F 31 r$, were spread on single EMA medium plates (eMA medium for $C$-30) which, after drying, were stored overnight in the refrigerator. Loop inocula from each culture and a similar culture of wild-type were also spread over well-separated circular areas of about $1 \mathrm{~cm}$. diameter on each of 6 TMA plates, over a template. After overnight incubation, replication was carried out (Lederberg \& Lederberg, 1952) from each of these plates to single different refrigerated lawn plates and to an additional uninoculated plate. These plates were then observed during incubation for $96 \mathrm{hr}$.

Table 2. Syntrophism of representative met mutants of Salmonella typhimurium

Samples $(0.1 \mathrm{ml}$.) of washed organisms from overnight broth cultures of each mutant were spread as lawns on single EMA medium plates $(e M A$ medium for metC-30) which were refrigerated overnight. Loop inocula of each mutant and wild-type were spread over areas about $1 \mathrm{~cm}$. diameter on each of 6 TMA medium plates, over a template. After overnight incubation, replication was made from each of these plates to a different refrigerated plate and to an uninoculated EMA medium plate. These plates were then incubated for at least $96 \mathrm{hr}$.

$\begin{array}{lccccr}\text { Replicate areas } & \text { met } A-15 & B-23 & C-30 & E-47 & F-31 r \\ \text { met } A-15 & -, 2 & -, 6 & -, 6 & -, 6 & -, 6 \\ \text { metB-23 } & +, 2 & -, 2 & -, 6 & -, 6 & -, 6 \\ \text { metC-30 } & +, 4 & +, 2 & -, 5 & -, 6 & -, 7 \\ \text { metE-47 } & \pm, 6 & -, 6 & \pm, 7 & -, 1 & -, 2 \\ \text { met } F-31 r & \pm, 6 & -, 5 & \pm, 6 & -, 1 & -, 1 \\ \text { Wild-type } & -, 8 & -, 8 & \pm, 8 & -, 8 & -, 8\end{array}$

$+=$ presence of halos of growth around replicated area; $-=$ absence of halos of growth around replicated area; $\pm=$ poor halos of growth around replicated area; $1-8$, relative density of growth within replicated area.

Syntrophism was evident from the growth response of organisms in the surface lawns as halos around replicated areas, and from the relative density of growth within different replicated areas on each lawn (Table 2). Halos indicated the diffusion of an accumulant to which organisms in the lawns responded. Thus it was inferred that the metabolic block of strain met $A-15$ preceded that of $B-23$ and that both the met $A-15$ and $B-23$ blocks preceded that of metC-30. There was also a suggestion that the metA-15 and $C$-30 blocks preceded those of metE-47 and $F-31 r$. Allowing for poorer growth within areas surrounded by halos, and the leaky growth within $m e t C-30$ areas, the relative density of growth within other areas confirmed the inferred sequence of metabolic blocks metA-15-metB-23-metC-30. No syntrophism of met $E-47$ and $F-31 r$ was observed, but syntrophism of metA-15, B-23 and $C$-30 with both metE-47 and $F-31 r$ did occur, although the direction of feeding was uncertain. Fourteen of the other 25 (met $A$ and met $B$ ) mutants behaved exactly as met $A-15$ and 11 exactly as met-B23 in further similar experiments. This indicated that these mutants might be divided into two phenotypic groups, comprising 15 met $A$ and 12 met $B$ mutants.

To find the direction of feeding in syntrophism of met $A$, metB or metC mutants with metE or metF mutants, the relative numbers of organisms of each phenotype in replicate areas showing syntrophism of metA-15, metB-23 or metC-30 with metE-47 or metF-31i was determined, assuming that fed organisms would pro- 
liferate more than feeders. Loopfuls of growth from these areas were suspended in $0.5 \mathrm{ml}$. phosphate buffer and appropriate dilutions plated on TMA medium to yield about 100 single colonies/plate. After overnight incubation replication to MA, MA + homocysteine thioloactone, MA + vitamin $\mathbf{B}_{12}$ (where metE organisms were involved) and TMA media was carried out and the plates incubated for $4 \mathrm{hr}$. at $37^{\circ}$ and $17 \mathrm{hr}$. at room temperature.

Between 200 and $\mathbf{5 0 0}$ colonies derived from each area were screened. In all cases, colonies with the $m e t A, B$ or $C$ phenotype predominated. They comprised an average of $91 \%$ of those from organisms in areas on lawns of metA-15, met-B23 or metC-30 and $82 \%$ from areas on lawns of metE-47 or metF-31r. It was concluded that the met $A$, met $B$ and met $C$ metabolic blocks preceded those of metE and met $F$.

In summary, the results of the syntrophism tests indicated the existence of 5 phenotypic groups amongst the methionineless mutants $($ met $A, \operatorname{met} B, \operatorname{met} C, \operatorname{met} E$, $m e t F)$ and that the probable sequence of the metabolic blocks in the biosynthesis of methionine was: met $A-$ metB-metC-(metE-metF). The arrangement of the metE and met $F$ blocks in sequence was not possible because of the lack of syntrophism of metE and metF mutants.

\section{The relationship between phenotypic and transduction groups}

This was tested by determining the frequency of transduction to produce prototrophs between all mutants of each phenotypic group and single representative mutants of other groups as recipients with appropriate single representative strains of each of Glover's $(1956,1958)$ five groups and the wild-type as donors. A lower frequency of transduction between mutants indicated close linkage and membership of the same transduction group (Demerec, Blomstrand \& Demerec, 1955). The experiments were carried out in three stages.

The transduction groups amongst the met $\boldsymbol{A}$ and met $B$ mutants were determined by using the original transduction technique. The selective medium was EMA. From the results recorded in Table $3(a)$ it can be seen that relatively few if any prototrophs resulted from transduction between all 15 of the met $A$ mutants and met $A-6$, or between the 12 met $B$ mutants and met $B-17$, thus indicating two transduction groups.

Transduction involving all of the metC strains as recipients was carried out in T2 buffer instead of broth; plating was on eMA medium. This controlled residual growth of the leaky metC strains and made prototrophic colonies easier to count. The improved transduction technique was used. The results in Table $\mathbf{3}(b)$ indicated that 9 of the 10 metC mutants comprised one transduction group. A high frequency of reversion did not permit the use of $m e t C-57$ as a recipient or donor.

Transduction groups amongst the met $E$ and met $F$ mutants were determined at the same time. Of the three metF mutants ('Table 1), metF-59 and metF-64 showed high frequencies of reversion. That of $m e t F-59$ was too high for its use as donor or recipient but that of metF-64 (approximately 1 in $1.25 \times 10^{6}$ organisms) permitted its use as a donor. The improved transduction technique was used, with plating on MA medium. All of the metE mutants appeared in one group and metF-31 and metF-64 in another (Table $3(c)$ ). The decreased frequency of transduction in the metB-23 $(\times)$ metF-64 cross may indicate linkage between these two mutants. Although all possible crosses between methionineless mutants of the same 
Table 3. The numbers of prototrophs obtained in transduction between Salmonella typhimurium met mutants of different phenotypic groups

Each figure represents the total number of colonies obtained on three enriched MA plates (EMA or eMA media) less the total number of colonies on three recipient control plates after $41 \mathrm{hr}$. of incubation. Each plate was inoculated with about $2 \times 10^{8}$ organisms from overnight broth cultures (centrifuged and resuspended in T2 buffer where metC mutants were recipients) and 1 or $4 \times 10^{9}$ phage particles which had been incubated together for $6 \mathrm{~min}$.

(a) met $A$ and metB mutants

\begin{tabular}{|c|c|c|c|c|c|c|c|}
\hline \multirow[b]{2}{*}{ Recipient } & \multicolumn{3}{|c|}{ Donor } & \multirow[b]{2}{*}{ Recipient } & \multicolumn{3}{|c|}{ Donor } \\
\hline & $\operatorname{met} A-6$ & metB-17 & $\begin{array}{l}\text { Wild- } \\
\text { type }\end{array}$ & & met $A-6$ & metB-17 & $\begin{array}{l}\text { Wild } \\
\text { type }\end{array}$ \\
\hline $\operatorname{met} A-6$ & 0 & 379 & 211 & $m e t B-16$ & 81 & $\mathbf{0}$ & 128 \\
\hline $4-7$ & $\mathbf{0}$ & 175 & 122 & $B-17$ & 115 & 0 & 114 \\
\hline$A-10$ & 0 & 476 & 240 & $B-20$ & 83 & 0 & 156 \\
\hline $.1-15$ & $\mathbf{0}$ & 41 & 38 & $B-23$ & 140 & $\mathbf{5}$ & 138 \\
\hline A-18 & $\mathbf{0}$ & 120 & 62 & $B-33$ & 213 & o & 195 \\
\hline$A-19$ & 10 & 81 & 44 & $B-34$ & 91 & $\mathbf{0}$ & 111 \\
\hline$A-21$ & 2 & 148 & 81 & B-36 & 212 & 0 & 308 \\
\hline A-22 & 3 & 109 & 82 & $B-39$ & 93 & 9 & 147 \\
\hline$A-40$ & 7 & 419 & 170 & $B-41$ & 72 & 0 & 121 \\
\hline$A-43$ & $0^{\circ}$ & 445 & 268 & $B-45$ & 168 & 26 & 201 \\
\hline$A-44$ & 19 & 210 & 102 & $B-49 *$ & 20 & 0 & 14 \\
\hline $4-48 *$ & 2 & 125 & 64 & $B-66^{*}$ & 142 & 21 & 176 \\
\hline A-53* & $\overline{3}$ & 146 & 83 & $C-32$ & 96 & 259 & 137 \\
\hline$A-54^{*}$ & 4 & 139 & 74 & $E-2$ & 91 & 92 & 86 \\
\hline$A-55^{*}$ & o & 143 & 65 & $F-3 I$ & 110 & 213 & 142 \\
\hline
\end{tabular}

* New mutants assigned to their transduction groups.

(b) metC mutants

\begin{tabular}{|c|c|c|c|c|c|}
\hline \multirow[b]{2}{*}{ Recipient } & \multicolumn{2}{|c|}{ Donor } & & \multicolumn{2}{|c|}{ Donor } \\
\hline & metC-30 & Wild-type* & Recipient & $m e t C-30$ & Wild-type* \\
\hline metC-30 & 0 & 3840 & $\operatorname{met} C-57$ & High frequency & f reversion \\
\hline$C-32$ & 590 & 5850 & $C-58$ & $\mathbf{0}$ & 1445 \\
\hline$C-35$ & 207 & 6705 & $C .65$ & 9 & 5245 \\
\hline$C-37$ & 72 & 2950 & $4-15$ & 3554 & 1615 \\
\hline$C-50$ & 24 & 1725 & B-23 & 4040 & 3050 \\
\hline$C-52$ & 721 & 3255 & $E-2$ & 3977 & 2955 \\
\hline$C-56$ & 712 & 3115 & $F-31$ & 6135 & 5615 \\
\hline
\end{tabular}

* Counts of platings of $1 / 5$ dilutions $\times 5$.

(c) metE and metF mutants

\begin{tabular}{|c|c|c|c|c|}
\hline Recipient & metE-2 & metF-31 & metF-64 & Wild-type* \\
\hline metE-2 & o & 6459 & 3719 & 4180 \\
\hline$E-47$ & 798 & $\mathbf{X}$ & $\mathbf{x}$ & 6910 \\
\hline$E-51$ & 38 & $\mathbf{X}$ & $\mathbf{X}$ & 695 \\
\hline$E-60$ & 295 & $\mathbf{X}$ & $\mathbf{x}$ & 4970 \\
\hline$E-61$ & 124 & $\mathbf{x}$ & $\mathbf{x}$ & 2310 \\
\hline$E-68$ & 108 & $\mathbf{X}$ & $\mathbf{X}$ & 5720 \\
\hline$F-31$ & $11010 *$ & 0 & 604 & 8710 \\
\hline$A-15^{*}$ & 7170 & 8630 & 6550 & 5910 \\
\hline$B-23^{*}$ & 6190 & 5320 & 1750 & 5800 \\
\hline$C-30^{*}$ & 3920 & 5010 & 2790 & 3840 \\
\hline
\end{tabular}


phenotypic groups were not made, the results of the above experiments were considered to show that the transduction and phenotypic grouping of these mutants was coincident.

\section{Attempts to detect abortive transduction}

Attempts to detect abortive transduction between representative mutants of each met group and wild-type were carried out at different times in several different ways. Both mid and late log-phase recipient cultures were used. Plating from T2 buffer as well as broth to MA, eMA, EMA, MA + neutralized vitamin-free casein hydrolysate at $\mathbf{0 \cdot 2} \%$ and $\mathrm{MA}+$ casamino acid at $0 \cdot 1 \%$ (Demerec et al. 1957) media was tested. Observations were made during incubation for 7 days, either continuously at $37^{\circ}$ or for 1 day at $37^{\circ}$ and then 6 days at room temperature as suggested by $\mathrm{Dr} \mathrm{H}$. Ozeki (personal communication). In no case were colonies characteristic of abortive transduction obtained (Ozeki, 1956) although Ozeki (1959) reported abortive transduction in the cross metC-50 $(\times)$ wild-type.

\section{Mapping the sites of mutation of the metB mutants}

This was attempted by determining the frequency of transduction to produce prototrophs in a series of crosses between them. The improved transduction technique was used and plating was on EMA medium. The same phage preparations were used throughout and estimates of donor and recipient efficiency obtained by transductions using $c y s C-7$ as recipient with all the metB strains as donors, and all of these strains as recipients with phage from the wild-type donor strain. To facilitate counting, $1 / 10$ broth dilutions of these control transduction mixtures were plated. From the results (Table 4) three transduction subgroups within which

Table 4. The numbers of prototrophs obtained in transductions between Salmonella typhimurium mutants of the metB group

Numbers as in Table 3. Plating was on EMA medium.

Recipient

Donor

\begin{tabular}{|c|c|c|c|c|c|c|c|c|c|c|c|c|c|}
\hline & \multicolumn{12}{|c|}{ metB } & \multirow{2}{*}{$\begin{array}{c}\text { Wild-* } \\
\text { type }\end{array}$} \\
\hline & -16 & -17 & -20 & -23 & -41 & -66 & -33 & -34 & -49 & -36 & -39 & -45 & \\
\hline metB-16 & 0 & 0 & 0 & 314 & 613 & 393 & $30 \%$ & 239 & 132 & 0 & 453 & 542 & 7170 \\
\hline-17 & 0 & 0 & 0 & 277 & 404 & 392 & 363 & 155 & 118 & 0 & 444 & 457 & 7040 \\
\hline-20 & 0 & 0 & 0 & 56 & 20 & 66 & 110 & 8 & 44 & 0 & 69 & 137 & 9200 \\
\hline-23 & 267 & 218 & 33 & 0 & 9 & 11 & 161 & 73 & 123 & 0 & 241 & 78 & 6300 \\
\hline-41 & 299 & 126 & 80 & 13 & 0 & 18 & 168 & 35 & 96 & 0 & 156 & 157 & 4610 \\
\hline-66 & 248 & 144 & 87 & 6 & $2 \pi$ & 0 & 153 & 40 & 98 & 0 & 81 & 147 & 4930 \\
\hline-33 & 379 & 192 & 132 & 115 & 220 & 256 & 0 & 44 & 0 & 0 & 135 & 541 & 8230 \\
\hline-34 & 125 & 112 & 0 & 36 & 28 & 130 & 21 & 0 & 0 & 0 & 147 & 240 & 9220 \\
\hline-49 & 1073 & 466 & 140 & 322 & 475 & 622 & 0 & 35 & 0 & 0 & 212 & 856 & 10260 \\
\hline-36 & 0 & 0 & 0 & 0 & 0 & 0 & 0 & 0 & 0 & 0 & 0 & 0 & 1350 \\
\hline-39 & 360 & 179 & 221 & 159 & 153 & 158 & 646 & 67 & 56 & 0 & 0 & 484 & 8580 \\
\hline-45 & 1233 & 535 & 532 & 136 & 260 & 129 & 975 & 265 & 316 & 0 & 578 & 0 & 13040 \\
\hline cys $C-7^{*}$ & 14710 & 12570 & 5210 & 4990 & 7020 & 16880 & 5760 & 4780 & 4950 & 15250 & 6590 & 6890 & 13380 \\
\hline
\end{tabular}


transduction occurred at low frequency or not at all were apparent. They comprised metB-16, metB-17 and metB-20; metB-23, metB-41 and metB-66; metB-33, $m e t B-34$ and $m e t B-49$, respectively. No transduction occurred between the members of the first sub-group or between metB-33 and metB-49 in the third subgroup. On the basis of frequency of reversion and behaviour in crosses with all other met $B$ mutants, metB-16 and metB-17 were similar to each other but different from $m e t B-20$. However, $m e t B-33$ and $m e t B-49$ were dissimilar in these respects.

No transduction was obtained in reciprocal crosses between metB-36 and all other metB mutants, and transduction between $m e t B-36$ and wild-type occurred at a lower frequency than that between other metB mutants and wild-type. As a donor in crosses with mutants of other met groups, metB-36 was as efficient as other $m e t B$ mutants. No reversions were detected on met $B-36$ control plates even when each of 10 plates was inoculated with 10 times the number of organisms normally plated in transduction experiments.

The construction of a linkage map from the data of Table 4 was attempted. To correct for donor and recipient efficiency, the frequency of transduction for each cross was multiplied by both the ratios of the frequencies of transduction in the crosses $c y s C-7(\times)$ wild-type/cysC-7 $(\times)$ donor, and $c y s C$ - $7(\times)$ wild-type/recipient $(x)$ wild-type, respectively. As an initial step, linear arrangement of the three transduction subgroups was tried. From the results of an analysis of corrected data from reciprocal crosses between different sets of single representative mutants of each subgroup it was not possible to construct a linear map. The same difficulty was encountered by using the sum of reciprocal transduction between pairs of met strains (after Hartman, 1956).

\section{Linkage between the metA, B, C, E and $\mathrm{F}$ groups}

This was tested by transduction between the representative strains met $A-15$, $B-23, C-30, E-47$ and $F-31$ in a series of donor phenotype selection experiments similar to those used by Clowes (1958) with cyst(e)ineless mutants. If donor and recipient differ phenotypically and it is possible to detect the donor phenotype amongst large numbers of organisms of the recipient phenotype, then the frequency with which transduction between mutants results in organisms which are wild-type for the recipient marker but mutant for the donor marker, should indicate the proximity of the sites of mutation concerned.

Three donor phenotypes were potentially detectable in transduction between pairs of mutants belonging to the different met groups. They were: the ability to utilize homocysteine thiolactone $\left(m e t^{\text {homth }}\right)$ or vitamin $\mathrm{B}_{12}\left(m e t^{\mathrm{B}_{13}}\right)$ as an alternative to methionine; attributes of met $A$, met $B$ or metC and met $E$ mutants, respectively; and leaky growth on MA medium when plated from broth $\left(\right.$ met $\left.^{\text {leaky }}\right)$ coupled with feeding of met $A$ and metB mutants, attributes of met $C$ mutants. The crosses in which each phenotype might have been detected are indicated in Table 5.

The ability to differentiate colonies with the different met phenotypes from wildtype arising after transduction was established in a series of reconstruction experiments under the conditions summarized in Table 6. All the permissible types of crosses (Table 5) were made by using the improved transduction technique, suitable dilutions of the transduction mixtures being made to give 100-200 transduced colonies/ plate. Infected recipient cultures of metC-30 were diluted in $\mathrm{T} 2$ buffer rather than 
in broth, to avoid too heavy background growth on the selective medium. Approximately 2000 (range 1257-3025) colonies from each cross were screened for donor phenotype. Transduction of donor phenotype was detected only in the metF-31 $(\times)$ met $B$ - 23 cross in which 1139 out of $3025(38 \%)$ transduced colonies were of the $m e t^{\text {homth }}$ phenotype.

In these experiments, linkage between all pairs of mutants from the different groups, except between those of met $A$ and $m e t B$, was tested in crosses involving transduction in at least one direction. Linkage between met $A$ and metE, metB and $m e t E$ and $m e t C$ and $m e t E$ was tested in both directions (Table 5). It was concluded that no linkage was detectable except between $m e t B$ and $m e t F$. Assuming a linear arrangement of sites of mutation and linkage of met $B$ and met $F$, met $A$ could either be between $m e t B$ and $m e t F$ or distal to $m e t B$ on the same side of met $F$ as metB. If the first situation prevailed, greater than $38 \%$ linked transduction between $m e t A$ and $m e t F$ should have been obtained. In fact no met $A$-met $F$ linkage was detected. Allowing for a $2 \%$ error in the replication technique (Clowes, 1958) and assuming that the production of transducing phage did not involve fragmentation between $m e t A$ and $m e t B$ (Ozeki, 1959), it was concluded that, if $m e t A$-met $B$ linkage existed, the distance between met $A$ and met $B$ was at least 19 times that between met $B$ and metF.

Table 5. Donor phenotypes Salmonella typhimurium met in mutants detectable in inter-group transduction

Recipient

Donor (phage)

\begin{tabular}{|c|c|c|c|c|c|}
\hline & met $A$ & $B$ & C & $E$ & $\boldsymbol{F}$ \\
\hline met $A$ & 0 & $\mathbf{x}$ & $m e t^{\text {leaky }}$ & $m e t^{\mathbf{B}_{19}}$ & $\mathbf{x}$ \\
\hline$B$ & $\mathbf{x}$ & 0 & met leaky & $m e t^{\mathrm{B}_{12}}$ & $\mathbf{x}$ \\
\hline$C$ & $\mathbf{x}$ & $\mathbf{x}$ & 0 & $m e t^{\mathbf{B}_{18}}$ & $\mathbf{x}$ \\
\hline $\boldsymbol{E}$ & $m e t^{\text {thomth }}$ & $m e t^{\text {homth }}$ & $m e t^{\text {homth }}$ & 0 & $\mathbf{x}$ \\
\hline$F$ & $m e t^{\text {homth }}$ & $m e^{\text {homth }}$ & $m e e^{\text {homth }}$ & $m e t^{\mathrm{B}_{12}}$ & 0 \\
\hline
\end{tabular}

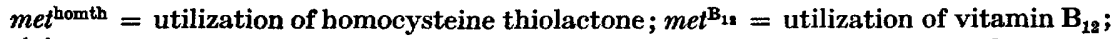
$m^{\text {eleaky }}=$ leaky growth on MA and feeding of $m e t A$ and $B$ strains; $\mathrm{X}=$ donor phenotype not detectable; $0=$ homologous crosses.

In conjunction with the previously described attempts to map the sites of mutation of metB mutants (Table 4) the frequency of transfer of donor phenotype in crosses between metF-31 and each of the metB mutants was determined. Transduction was carried out under optimum conditions for the detection of the metB met $^{\text {homth }}$ phenotype (Table 6) except that only about 1000 colonies/cross were screened for donor phenotype. An average of $27 \cdot 5 \%$ (range $21 \cdot 6-34 \cdot 1 \%$ ) transfer of donor phenotype was obtained in crosses involving 11 of the 12 metB mutants as donors. The exception was metB-36 with which only $3 \cdot 6 \%$ transfer was obtained. These results indicated that all the metB sites were linked to metF-31 but that 11 of them were clustered together closer to metF-31 than to metB-36.

\section{Linkage between met mutants and certain other markers}

Linkage was tested by transduction in two ways.

By donor phenotype selection. This was carried out in transduction between the 5 representative met mutants used in the intergroup crosses above (recipients) and the six double-auxotrophs metA-7cysA-21, metB-16cysB-16, metE-2cysC-7, 
Table 6. Optimum conditions for the detection of the different donor phenotypes of met mutants of Salmonella typhimurium in intergroup transduction

\begin{tabular}{|c|c|c|c|c|c|c|}
\hline \multirow[b]{2}{*}{$\begin{array}{c}\text { Donor } \\
\text { phenotype }\end{array}$} & \multirow[b]{2}{*}{ Recipient } & \multicolumn{2}{|c|}{ Initial selection } & \multicolumn{2}{|c|}{ Replication } & \multirow[b]{2}{*}{$\begin{array}{c}\text { Characteristics } \\
\text { of donor } \\
\text { phenotype }\end{array}$} \\
\hline & & Medium & $\begin{array}{l}\text { Time of } \\
\text { incuba- } \\
\text { tion }\end{array}$ & Media & $\begin{array}{l}\text { Time of } \\
\text { incuba- } \\
\text { tion }\end{array}$ & \\
\hline $\begin{array}{l}m e t^{\text {homth }} \\
(m e t A, B \text { and } \\
C)\end{array}$ & $\begin{array}{l}m e t E \text { and } \\
F\end{array}$ & EMA + homth & $41 \mathrm{hr}$. & $\begin{array}{l}\text { MA and } \\
\text { MA +homth }\end{array}$ & $17 \mathrm{hr}$. & $\begin{array}{l}\text { Growth on MA }+ \\
\text { homth; not on MA }\end{array}$ \\
\hline$m e t^{\text {leaky }}(m e t C)$ & $\underset{B}{\operatorname{met} A} \operatorname{and}$ & MA & 6 days & • & • & $\begin{array}{l}\text { Microcolonies (1- } \\
1.5 \text { mm. diam.) } \\
\text { + feeding of back- } \\
\text { ground }\end{array}$ \\
\hline$m e t^{\mathrm{B}_{13}}(m e t E)$ & $\begin{array}{l}\text { met } A \text { and } B \\
\text { met } F \\
m e t C\end{array}$ & $\begin{array}{l}\mathbf{E M A}+\mathbf{B}_{12} \\
\mathbf{E M A}+\mathbf{B}_{12} \\
\mathbf{M A}+\mathbf{B}_{12}\end{array}$ & $\left.\begin{array}{l}4.1 \mathrm{hr} . \\
28 \mathrm{hr} . \\
4,1 \mathrm{hr} .\end{array}\right\}$ & $\begin{array}{l}\text { MA and } \\
\mathbf{M A}+\mathbf{B}_{12}\end{array}$ & $17 \mathrm{hr}$. & $\begin{array}{l}\text { Growth on } \mathrm{MA}+\mathrm{B}_{12} \\
\text { not on } \mathrm{MA}\end{array}$ \\
\hline
\end{tabular}

homth $=$ homocysteine thiolactone at $100 \mu \mathrm{g} . / \mathrm{ml}$.

$\mathbf{B}_{12}=$ vitamin $\mathbf{B}_{12}$ at $0.01 \mu \mathrm{g} . / \mathrm{ml}$. (i.e. 1/10th of concentration used in growth response experiments)

met $B-20$ try $A-8$, met $F-31$ adth $A-2$ and met $B-41$ ade $E-11$ (donors). The initial selective medium was supplemented with cysteine, tryptophan, adenine and thiamin or adenine where appropriate, to facilitate growth of colonies with donor phenotype. The screening of about 1000 colonies from each cross was attempted. Initial selection plates were incubated for 24-65 hr. according to the cross, until all of the transduced colonies were about the same size (1-2 mm. diameter) before replication to unsupplemented and supplemented media. The replicated colonies were observed

Table 7. Attempts to detect the transfer of donor phenotype in transduction between representative mutants of the five met groups and certain other mutants of Salmonella typhimurium

Transduction was as described in Table 3. The MA medium for each cross was supplemented with either cysteine, tryptophan, adenine or adenine + thiamin according to the additional nutritional requirement of each donor strain. The initial incubation was for 24-65 hr. After replication to unsupplemented and supplemented MA medium the plates were incubated for $17 \mathrm{hr}$.

Recipient

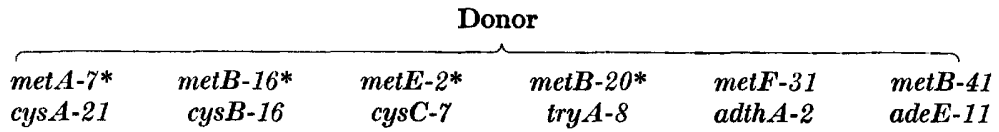

No. of colonies screened without detecting donor phenotype

\begin{tabular}{rrrrrrr} 
met $A-15$ & \multicolumn{1}{c}{0} & 1197 & 1107 & 1024 & 1060 & 1034 \\
$B-23$ & 1140 & 841 & 730 & 0 & 1047 & 0 \\
$C-30$ & 603 & 1279 & 716 & 1056 & 975 & 1288 \\
$E-47$ & 1355 & 1533 & 941 & 1132 & 851 & 820 \\
$F-31$ & 1665 & 1401 & 1316 & 847 & $\times$ & $* *$
\end{tabular}

* No linked transduction between these double auxotrophs and wild-type (see Table 8 ).

** Not tested, since selection of donor phenotype ade impossible because the additional marker of $m e t F-31$ is $a d t h$.

$\times$ Homologous cross. 
after incubation for $17 \mathrm{hr}$. No colonies with donor phenotype resulted from any of these crosses (Table 7). Three crosses yielded no transduction and the donor phenotype could not be selected in another so that linkage between met $A$ and cys $A$, $m e t B$ and $\operatorname{try} A$ or adeE and metF and adthA could not be tested. There therefore appeared to be no linkage between: $m e t A$ and $c y s B, \operatorname{cys} C, \operatorname{try} A, \operatorname{adth} A$ and adeE; between $m e t B$ and $c y s A, c y s B, c y s C$ or $a d t h A$; between met $C$ and $c y s A, c y s B, c y s C$, $\operatorname{try} A, \operatorname{adth} A$ or adeE; between met $E$ and $c y s A, \operatorname{cys} B, c y s C, \operatorname{try} A, \operatorname{adth} A$ or adeE; or between met $F$ and $c y s A, c y s B, c y s C$ or $\operatorname{try} A$.

$B y$ transduction of doubly auxotrophic recipients with phage from wild-type donor

This involved the attempted transduction of both markers simultaneously. Crosses of all of the double auxotrophs used above, with metA-19 tryA-8 substituted for met $F-31$ adth $A-2$, were made by the improved transduction technique. Each transduction mixture was plated on MA medium, MA + methionine medium and MA + cysteine, tryptophan or adenine medium, according to the alternative nutritional requirement of the recipient. Colonies on the supplemented media were counted after incubation for $41 \mathrm{hr}$. Final observations of all plates were made after incubation for a further $48 \mathrm{hr}$. Replication was then made from the supplemented plates to MA medium. The replica plates were observed for wild-type colonies during incubation for $48 \mathrm{hr}$. The results of these experiments (Table 8), confirmed the absence of linkage between $m e t A$ and $\operatorname{try} A$, met $B$ and $c y s B$ and $m e t E$ and $c y s C$, and also indicated its absence between met $A$ and $c y s A$ and met $B$ and $\operatorname{try} A$ or adeE.

Table 8. Attempts to detect linked transduction between certain Salmonella typhimurium met mutants and other loci, by the use of double auxotrophs

\begin{abstract}
Numbers as in Table 3 except that plating was on MA medium. Where the MA medium was supplemented, platings were made from $1 / 10$ dilutions of the transduction mixtures in addition to those from the concentrated suspensions.
\end{abstract}

\begin{tabular}{|c|c|c|c|c|c|}
\hline \multicolumn{6}{|c|}{ Recipient } \\
\hline $\begin{array}{c}\operatorname{met} A-7 \\
\operatorname{cus} A-21\end{array}$ & $\begin{array}{c}\text { metB-16 } \\
\text { cysB-16 }\end{array}$ & $\begin{array}{l}\text { metE-2 } \\
\text { cysC-7 }\end{array}$ & $\begin{array}{c}\operatorname{met} A-19 \\
\operatorname{try} A-8\end{array}$ & $\begin{array}{c}\text { metB-20 } \\
\operatorname{tr} y A-8\end{array}$ & $\begin{array}{l}\text { metB-41 } \\
\text { adeE-11 }\end{array}$ \\
\hline
\end{tabular}

\begin{tabular}{|c|c|c|c|c|c|c|}
\hline \multirow[b]{2}{*}{$\begin{array}{l}\text { Media of } \\
\text { selection }\end{array}$} & \multicolumn{6}{|c|}{ No. of colonies resulting from transductions using wild type donor } \\
\hline & & & & & & \\
\hline MA & $\mathbf{0}$ & $\mathbf{0}$ & $\mathbf{0}$ & $\mathbf{0}$ & $\mathbf{0}$ & $\mathbf{0}$ \\
\hline $\begin{array}{l}\text { MA + methio- } \\
\text { nine }\end{array}$ & 4500* & 1484 & $3000 *$ & $9000^{*}$ & $14000^{*}$ & 1968 \\
\hline $\begin{array}{l}\text { MA + cysteine, } \\
\text { tryptophan or }\end{array}$ & $\begin{array}{r}544 \\
\text { denine }\end{array}$ & 1214 & 568 & $3580 *$ & 4070 & 2765 \\
\hline
\end{tabular}

\title{
DISCUSSION
}

\section{Metabolic blocks in a possible pathway of methionine biosynthesis}

Homocysteine is believed to be a precursor of methionine in several different micro-organisms, e.g. Neurospora crassa, Bacillus subtilis, Escherichia coli (Young \& Maw, 1958) and Streptococcus lactis (Kizer, Speck \& Aurand, 1955) and is implicated in the synthesis in Aerobacter aerogenes (Shapiro, 1955). Davis \& Mingioli (1950) reported methionineless mutants of $E$. coli W which showed similar growth 
responses and syntrophism to those of the Salmonella typhimurium metE and met $A$, $B$ and $C$ mutants, and postulated that vitamin $B_{12}$ was a component of a coenzyme concerned with the methylation of homocysteine. This function of vitamin $\mathbf{B}_{12}$ seemed to be supported by studies of the synthesis of methionine with cell suspensions and cell-free extracts of at least one of these mutants (Gibson \& Woods, 1952; Helleiner \& Woods, 1956). The growth response of members of the $S$. typhimurium met $A, B$ and $C$ phenotypic groups to homocystine and homocysteine thiolactone suggested that homocystine or homocysteine (homocyst(e)ine) was also on the pathway of methionine biosynthesis in $S$. typhimurium and that these mutants were blocked in metabolic steps before this intermediate, and metE and $F$ mutants after it. This sequence is supported by the results of the syntrophism experiments and the existence of three metabolic blocks associated with S. typhimurium met $A$, metB and metC mutants in that sequence established (Fig. 1).

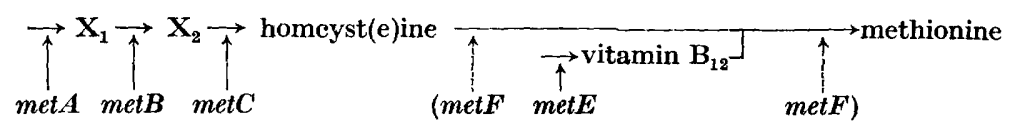

$\mathbf{X}=$ unknown precursor of methionine; $\hat{i}=$ possible alternative positions of metabolic block; ()$=$ indeterminate sequence of blocks.

Fig. 1. A suggested pathway of methionine biosynthesis in Salmonella typhimurium.

Since metE and metF mutants fed met $A, B$ and $C$ mutants but themselves failed to show syntrophism, it was concluded that their metabolic blocks involved inability to carry out some (probably the same) biosynthetic step between homocys$t(e)$ ine and methionine. It is suggested that the function of vitamin $B_{12}$ in the biosynthesis of methionine is the same in Salmonella typhimurium and Escherichia coli and that the $S$. typhimurium metE mutants lack the coenzyme concerned with the methylation of homocysteine of which vitamin $B_{12}$ (or a derivative of it) is a component. $S$. typhimurium metF mutants may be considered to lack the enzyme necessary for this metabolic step.

The results of these biochemical studies are in general agreement with those outlined by Glover $(1956,1958)$ who used some of the same mutants, except that the growth response of Salmonella typhimurium met $A$ and $B$ mutants to cystathionine was not clearly confirmed. However, cystathionine should not perhaps be excluded as an intermediate, for Wijesundera \& Woods (1953) showed that 8 out of $10 \mathrm{mu}$ tants of Escherichia coli which possessed cystathionase were able to convert exogenous cystathionine to homocysteine but only synthesized small quantities of methionine. They suggested that other enzymes might compete for homocysteine; perhaps the differences between cultural conditions for $\boldsymbol{S}$. typhimurium met $\boldsymbol{A}$ and $B$ mutants in Glover's experiments and those reported in this paper, affected the relative activity of these competing enzymes so that a definite growth response to cystathionine was obtained in the former but not in the latter.

\section{Correlation of phenotypic and transduction groups}

Members of the same transduction group of Salmonella typhimurium met mutants possessed identical biochemical characteristics so far as they were tested and each can be regarded as an allele of one of 5 complex gene loci (Demerec, 1956b) namely 
met $A$, met $B$, metC, metE and met $F$. In the absence of a complementation test (abortive transduction) it was not possible to decide whether each locus was a functional unit. Demerec \& Ozeki (1959) gave examples of complex loci defined in the same way as the met loci but subsequently shown to comprise more than one closely linked functional unit. They also reported, incidentally, that complementation might not always serve to differentiate such loci and that abortive transduction was not detectable with some mutants.

\section{The metB locus}

The attempts to map the sites of mutation of the metB locus in Salmonella typhimurium were subject to limitations, because, even with the improved transduction technique, the efficiency of transduction of met mutants was less than that of most other mutants of $\boldsymbol{S}$. typhimurium and hence the chances of recombination were decreased. The resolving power within met loci might well have been as low as $1 \%$ of that within the his locus (Hartman, Loper \& Serman, 1960) and 10\% of that within the try locus (Demerec \& Hartman, 1956) respectively. It was perhaps not surprising that a linear map of the metB locus could not be constructed. For accurate location of sites three- or four-point linkage tests are a great advantage, especially when the efficiency of transduction is low (compare Clowes's (1958) studies of the $c y s B$ locus). Unfortunately, no linkage was detected between any of the met loci and a number of others suitable for such tests.

The properties of Salmonella typhimurium metB-36 indicate that it is a multiplesite mutant (Demerec, 1956 $a$ ) in that it failed to recombine in reciprocal transduction with a number of closely-linked mutants, that it was the only mutant of the 12 metB mutants to do this, and that it failed to revert. Definition of the region of the chromosome covered by the metB-36 mutation is not possible at present. It does not cover the metF locus to which the metB locus is linked, but may cover an undiscovered locus (or loci) between $m e t B$ and $m e t F$ or on the other side of $m e t B$, or may even cover loci on both sides of metB. The failure to obtain transduction between one group of three (metB-16, B-17 and $B-20)$ and one pair (metB-33 and $B-49$ ) of the met $B$ mutants other than $m e t B-36$ may have been due to identical-site mutation at two sites (Demerec, 1956a). There is evidence that some identical-site mutants show similar frequencies of reversion (Demerec, 1956a), and similar behaviour in intra-locus transduction might be anticipated. For metB-16, B-17 and $B-20$ and $m e t B-33$ and $B-49$ incidental observations of their reversion frequency and the data of Table 4 revealed that only metB-16 and $B-17$ were similar in these respects. It was concluded that the sites of mutation of metB-16 and $B-17$ might well be identical, but metB-20 differed from these two and metB-33 and $B-49$ from each other, in that mutation in these mutants involved overlapping but probably different numbers of sites on the chromosome. The speculation could be made that each mutation in the metB locus other than metB-36 (probably a deletion) is a multiple-site change involving different small lengths of the chromosome. This would perhaps explain mapping difficulties within the locus.

\section{Behaviour of metB-36 in transduction}

In the met $F-31(\times)$ metB-36 cross only $3 \cdot 6 \%$ donor phenotype transfer was obtained as compared with an average of $27.5 \%$ with other met $B$ mutants as donors. 
From the results of transduction between all the metB mutants (Table 4), metB-36 appears to be a multiple-site mutant, probably a deletion. Interference with synapsis seems the most likely explanation of the decreased frequency of donor phenotype transfer with metB-36 and, incidentally, the decreased frequency of transduction in the cross $m e t B-36(x)$ wild-type in Table 4. In the former case this could have resulted from a reduction in size of the donor transducing fragments. If fragmentation of the donor chromosome in the preparation of phage lysates is not random (Ozeki, 1959), the formation of chromosomal fragments from met $B-36$ carrying the $m e t B$ and $F$ loci should include a deletion and therefore be shorter.

\section{Linkage relationships of the met loci}

The linkage between two of the 5 met loci is a further example of linkage between loci concerned with related function in Salmonella typhimurium (Demerec, 1956a). Detection of linkage of other met loci may have been impossible because of fragmentation of the donor chromosome between them in the preparation of transducing phage (Ozeki, 1959). In some instances the linear linkage sequence of functionally related loci of $S$. typhimurium was the same as that of the sequence of metabolic blocks associated with each locus, i.e. an 'assembly line' was formed. Five of the 7 loci concerned with histidine (Hartman, Loper \& Serman, 1960) and all the 4 loci concerned with tryptophan biosynthesis (Demerec \& Hartman, 1956) were shown to be arranged like this. Tryptophan biosynthesis in Escherichia coli involves the same pathway and is controlled by similarly linked loci (Yanofsky \& Lennox, 1959). Examples of less perfect associations prevail amongst the cysteine (Clowes, 1958) and proline loci (Demerec et al. 1958) of S. typhimurium, where one pair out of 5 and one pair out of 4 loci, respectively, were linked and associated with consecutive metabolic steps. In any case, with the possible exception of the purineless mutants (Yura, 1956a; Demerec et al. 1956), whenever linkage between functionally related loci was demonstrated, the equivalent metabolic blocks appeared to be consecutive (Demerec et al. 1955, 1958). The sequence of metabolic blocks in the biosynthesis of methionine is metA-metB-metC-(metE metF) so that the linkage of the $m e t B$ and $m e t F$ loci constitutes an example of linkage between functionally-related loci not concerned with consecutive metabolic blocks.

The selective advantage of the assembly line of genes has been stressed both from the functional (Demerec \& Demerec, 1955; Demerec \& Hartman, 1956) and genetical (Hartman, Loper \& Serman, 1960) points of view. Linkage between metB and $F$ indicates that at least two of the met loci are not arranged in an assembly-line sequence. However, within intact bacteria, folding and interfolding of the chromosome may ensure that functionally related loci are arranged in such a sequence (Pontecorvo, 1958). On the other hand, general acceptance of the genetical selective advantage of assembly lines to organisms with systems of partial genetic transfer should obviously be avoided.

Methionineless mutants of a number of different micro-organisms have been reported. However, appropriate biochemical and linkage data for comparison with the met mutants of Salmonella typhimurium are at present limited to strains of Escherichia coli, Neurospora crassa and Streptomyces coelicolor.

The information available on the methionineless mutants of Escherichia coli K 12 suggests similarities with Salmonella typhimurium (Clowes \& Rowley, 1954; 
Ruebner, 1956; Dr F. Jacob, personal communication). On the basis of growth responses, three phenotypic groups are recognizable. They are equivalent to the met $A+$ met $B+$ met $C$, the met $E$ and the met $\boldsymbol{F}$ mutants of $S$. typhimurium, respectively. In conjugation experiments one mutant of the first group (comprising 7 mutants) is linked to the only available representative of the last group (the M1 and M2 mutants of Clowes \& Rowley, 1954). The other 6 mutants of the first group are closely linked together and located some distance from M1 and M 2 on the circular linkage map of Pardee, Jacob \& Monod (1959). Mutants of the second group are closely linked together but unlinked to any of the other methionineless mutants. It is interesting to note that the only linkage between phenotypically different methionineless mutants of $E$. coli involves mutants similar to those of the $m e t B$ and $m e t F$ loci of $S$. typhimurium which were the only met loci of this organism shown to be linked.

Seven mutants of Neurospora crassa associated with at least four different blocks in methionine biosynthesis have been recognized. Six of these have been mapped (Barratt, Newmeyer, Perkins \& Garnjobst, 1954; Drs Catcheside \& Murray, personal communication). None of these is closely linked although three are located on the same chromosome. Hopwood (1959) recognized 4 loci concerned with methionine biosynthesis in Streptomyces coelicolor but none of these showed close linkage to the others. Three linkage groups were suggested; 2 of the loci were situated in one and one each in the other two.

The following linkage map for some loci of Salmonella typhimurium, including the 5 met loci, was suggested by Demerec \& Demerec $(1955)$ and Glover $(1955,1956)$ :

$$
\operatorname{try} D-\operatorname{try} C-\operatorname{try} B-\operatorname{try} A-c y s B-m e t E-c y s A-m e t C-m e t A-m e t B-m e t F-(c y s C \text { cys } D)
$$

From the transduction studies reported in the present paper, the inter-locus donor phenotype selection data support the suggestion of linkage between $m e t B$ and metF but not between metC and metA or met $A$ and metB. Failure to detect donor phenotype transfer in transduction involving certain double auxotrophs as donors (Table 7 ) and the absence of prototrophs results from transduction between certain double auxotrophs and wild type (Table 8 ), provide no evidence for linkage between $\operatorname{try} A$ and $m e t E, c y s B$ and $m e t E$, met $E$ and $c y s A, c y s A$ and $m e t C$ and $m e t F$ and $c y s C$. These results and those of similar experiments by Clowes (1958, and unpublished) do not support the linkage map of Demerec and his colleagues, at least for the region to the right of cysB.

This work was begun in the Department of Genetics, Carnegie Institution of Washington, Cold Spring Harber, Long Island, N.Y., U.S.A. during the tenure of an English Speaking Union King George VI Memorial Fellowship for 1955-6. I am indebted to Dr M. Demerec and his colleagues in this Department for the provision of mutants. I wish to thank Dr N. Horowitz (California Institute of Technology) for preparations of cystathionine and homocysteine thiolactone. At the Microbiological Research Establishment, Porton, thanks are due to Mr J. R. Slade for additional preparations of these compounds, to $\mathrm{Mr} \mathrm{H}$. E. Wade for carrying out chromotographic analyses and to Dr T. W. Burrows for helpful discussion. Much excellent technical assistance was given by Mr L. W. Stott. Some of this work formed part of a Ph.D. thesis submitted to the University of Reading in 1959. 


\section{REFERENCES}

Adams, M. H. (1950). Methods of study of bacterial viruses. Meth. med. Res. $2,1$.

Barrati, R. W., Newmeyer, D., Perkins, D. D. \& Garnjobst, L. (1954). Map construction in Neurospora crassa. Advan. Genet. 6, 1.

Clowes, R. C. (1958). Investigation of the genetics of cysteineless mutants of Salmonella typhimurium by transduction. J. gen. Microbiol. 18, 154 .

Clowes, R. C. (1960). Fine genetic structure as revealed by transduction. Symp. Soc. gen. Microbiol. 10, 92.

Clowes, R. C. \& Rowley, D. (1954). Some observations on linkage effects in genetic recombination in Escherichia coli K-12. J. gen. Microbiol. 11, 250.

DAvis, B. D. (1948). Isolation of biochemically deficient mutants of bacteria by penicillin. J. Amer. chem. Soc. 70, 4267.

Davis, B. D. \& Mingroli, E. S. (1950). Mutants of Escherichia coli requiring methionine or vitamin B12. J. Bact. 60, 17.

Demerec, M. (1956a). A comparative study of certain gene loci in Salmonella. Cold Spr. Harb. Symp. quant. Biol. 21, 113.

Demerec, M. (1956b). Terminology and nomenclature. Genetic studies with Bacteria. Publ. Carneg. Instn, 612, 1.

Demerec, M., Blomstrand, I. \& Demerec, Z. E. (1955). Evidence of complex loci in Salmonella. Proc. nat. Acad. Sci., Wash. 41, 359.

Demerec, M. \& Demerec, Z. E. (1955). Analysis of linkage relationships in Salmonella by transduction techniques. Brookhaven Symp. Biol. 8, 75.

Demerec, M., Goldman, I. \& Lahr, E. L. (1958). Genetic recombination by transduction in Salmonella. Cold Spr. Harb. Symp. quant. Biol. 23, 59.

Demerec, M., Hartman, P. E., Moser, H., Kanazir, D., Demerec, Z. E., Fitzgerald, P. L., Glover, S. W., Lahr, E. L., Westover, W. E. \& Yura, T. (1955). Bacterial Genetics. I. Carneg. Instn $\operatorname{Yr} B k, 54,219$.

Demerec, M. \& Hartman, Zlata (1956). Tryptophan mutants in Salmonella typhimurium. Genetics Studies with bacteria. Publ. Carneg. Instn, 612, 5.

Demerec, M., Lahr, E. L., Mryake, T., Goldman, I., Balbinder, E., Banič, S., Hashiмoto, K., Glanvilife, E. V. \& Gross, J. D. (1958). Bacterial genetics. Carneg. Instn, Yr $B k, 57,390$.

Demerec, M., Lahr, E. L., Ozeki, H., Goldman, I., Howarth, S. \& Djordjević, B. (1957). Bacterial Genetics. Carneg. Instn $\mathrm{Yr} B k$, 56, 368.

Demerec, M., Moser, H., Clowes, R. C., Lahr, E. L., Ozeki, H. \& Vielmetter, W. (1956). Bacterial Genetics. Carneg. Instn $\mathrm{Yr}$ Bk, 55, 301.

Demenec, M. \& OzekI, H. (1959). Tests for allelism among auxotrophs of Salmonella typhimurium. Genetics, 44, 279.

DENT, C. E. (1948). A study of the behaviour of some sixty amino-acids and other ninhydrin-reacting substances in phenol-'collidine' filter paper chromatograms with notes as to the occurrence of some of them in biological fluids. Biochem. J. 43, 169 .

DuBnoff, J. W. (1952). The role of B 12 in methionine synthesis in E. coli. Arch. Biochem. Biophys. 37, 37.

Grbson, F. \& Woons, D. D. (1952). The synthesis of methionine from homocysteine by Escherichia coli. Biochem. J. 51, v.

Glover, S. W. (1955). In Demerec et al. (1955).

Glover, S. W. (1956). Some aspects of the genetics of Escherichia coli and Salmonella typhimurium. Ph.D. thesis, University of Dublin.

Glover, S. W. (1958). A genetical and biochemical study of methionine mutants of Salmonella typhimurium. Proc. VIIth int. Con. Microbiol. 4 1., 57.

Gors, J. S. (1956). The biochemical nature of a suppressor mutation in a purine requiring mutant of Salmonella typhimurium. Genetic Studies with Bacteria. Publ. Carneg. Instn, 612, 87.

Hartman, P. E. (1956). Linked loci in the control of consecutive steps in the primary pathway of histidine synthesis in Salmonella typhimurium. Genetic Studies with Bacteria. Publ. Carneg. Instn, 612, 35. 
Hartman, P. E., Loper, J. C. \& Šrman, D. (1960). Fine structure mapping by complete transduction between histidine-requiring Salmonella mutants. J. gen. Microbiol. 22, 323.

Helleiner, C. W. \& Woods, D. D. (1956). Cobalamin and the synthesis of methionine by cell-free extracts of Escherichia coli. Biochem. J. 63, $26 \mathrm{P}$.

Hershey, A. D. \& Chase, M. (1953). Independent function of viral protein and nucleic acid in the growth of bacteriophage. J. gen. Physiol. 36, 39.

Hopwood, D. A. (1959). Linkage and the mechanism of recombination in Streptomyces coelicolor. Ann. N.Y. Acad. Sci. 81, 887.

Horowitz, N. H. (1947). Methionine synthesis in Neurospora. The isolation of cystathionine. J. biol. Chem. 171, 255.

Howarti, S. (1958). Suppressor mutations in some cysteine requiring mutants of Salmonella typhimurium. Genetics, 43, 404.

Kizer, D. E., Speck, M. L. \& Aurand, L. W. (1955). The effect of methionine and methionine precursors on the growth of Streptococcus lactis. J. Bact. 69, 16.

LEDERBERG, J. (1950). Isolation and characterization of biochemical mutants of bacteria. Meth. med. Res. 3, 5.

LederberG, J. \& Lederberg, E. M. (1952). Replica plating and indirect selection of bacterial mutants. J. Bact. 63, 399.

LederberG, J. \& Zinder, N. (1948). Concentration of biochemical mutants of bacteria with penicillin. J. Amer. chem. Soc. 70, 4267.

OzekI, H. (1956). Abortive transduction in purine-requiring mutants of Salmonella typhimurium. Genetic studies with bacteria. Publ. Carneg. Instn, 612, 97.

OzeKI, H. (1959). Chromosome fragments participating in transduction in Salmonella typhimurium. Genetics, 44, 457.

Pardee, A. B., Jacob, F. \& Monod, J. (1959). The genetic control and cytoplasmic expression of 'inducibility' in the synthesis of $\beta$-galactosidase by $E$. coli. J. mol. Biol. $1,165$.

Ponteconvo, G. (1949). Auxanographic techniques in biochemical genetics. J. gen. Microbiol. 3, 122.

Ponteconvo, G. (1958). Trends in Genetic Analysis. New York: Columbia University Press.

Ruebner, B. (1956). The biologic assay of L-methionine using a mutant of Bact. coli, strain K12. J. Lab. clin. Med. 47, 140.

Shapiro, S. K. (1955). The biosynthesis of methionine from homocysteine and methylmethionine sulphonium salt. Biochim. biophys. Acta, 18, 134.

Wijesundera, S. \& Woods, D. D. (1953). Cystathionine in relation to methionine synthesis by Bacterium coli. J. gen. Microbiol. 9, iii.

Yanofsky, C. \& Lennox, E. S. (1959). Transduction and recombination study of linkage relationships among the genes controlling tryptophan synthesis in Escherichia coli. V'ro$\log y, 8,425$.

Young, L. \& Maw, G. A. (1958). The Metabolism of Sulphur Compounds. London: Methuen and Co. Ltd.

YURA, T. (1956 $a)$. Evidence of non-identical alleles in purine-requiring mutants of Salmonella typhimurium. Genetic studies with bacteria. Publ. Carneg. Instn, 612, 63.

YurA, T. (1956b). Suppressor mutations in purine-requiring mutants of Salmonella typhimurium. Genetic Studies with Bacteria. Publ. Carneg. Instn, 612, 77.

Zinder, N. D. \& Lederberg, J. (1952). Genetic exchange in Salmonella. J. Bact. 64, 679. 